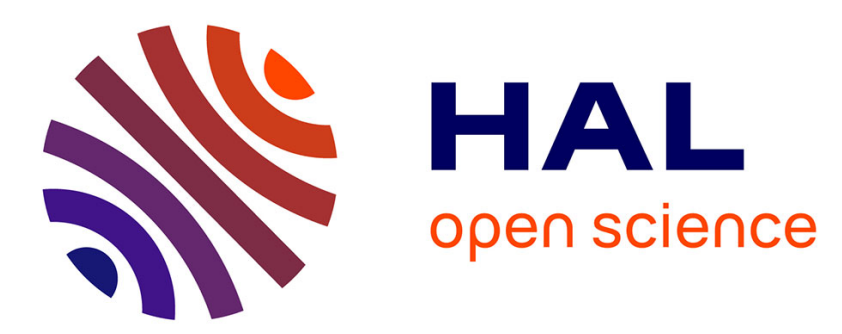

\title{
Symmetric-iterative solution of coupled BE and FE discretizations for elastoplasticity
}

Chunying Dong, Marc Bonnet

\section{To cite this version:}

Chunying Dong, Marc Bonnet. Symmetric-iterative solution of coupled BE and FE discretizations for elastoplasticity. Computer Methods in Applied Mechanics and Engineering, 1999, 178, pp.171-182. 10.1016/S0045-7825(99)00012-2 . hal-00092382

\section{HAL Id: hal-00092382 \\ https://hal.science/hal-00092382}

Submitted on 8 Dec 2018

HAL is a multi-disciplinary open access archive for the deposit and dissemination of scientific research documents, whether they are published or not. The documents may come from teaching and research institutions in France or abroad, or from public or private research centers.
L'archive ouverte pluridisciplinaire HAL, est destinée au dépôt et à la diffusion de documents scientifiques de niveau recherche, publiés ou non, émanant des établissements d'enseignement et de recherche français ou étrangers, des laboratoires publics ou privés. 


\title{
Symmetric-Iterative Solution of Coupled BE and FE Discretizations for Elastoplastics
}

\author{
C.Y. Dong ${ }^{a}$, Marc Bonnet ${ }^{b, *}$ \\ ${ }^{a}$ Department of Engineering Mechanics, Tsinghua University, Beijing 100084, People Republic of China \\ ${ }^{\mathrm{b}}$ Laboratoire de Mechanique des Solides, CNRS UMR 7649, Ecole Polytechnique, 91128 Palaiseau cedex, France
}

\begin{abstract}
In this paper the symmetric-iterative method of coupled FE and BE discretizations is adopted to investigate elastoplastic problems. In order to improve computational efficiency, all degrees of freedom related to the BE region, except those degrees of freedom associated with interface, are condenced. The symmetric part of the stiffness matrix from the boundary element region is assembled into the stiffness matrix of the FEM, but antisymmetric part is taken as the corresponding influential load matrix. During the elastoplastic solution process of the FEM, the numerical iterations which includes the influence of the antisymmetry of the condensed stiffness matrix are being carried out until the convergent results are obtained. Numerical examples are presented to illustrate the performance of the given algorithm and compared with the existing results.
\end{abstract}

Keywords: Boundary element method; Finite element method; Elastoplastic problems

\section{Introduction}

The combination of boundary element and finite element methods has been extensively investigated by many researchers and applied to various problems such as geomechanics, elastoplastic fracture mechanics, electromagnetics, acoustics etc. From the viewpoint of simplicity of application, we will only consider one of existing coupling methods, that is, the boundary element region is treated as a finite element. This approach can easily be incorporated into existing finite element codes.

The resulting stiffness matrix derived from conventional BEM formulation is the nonsymmetric and full populated. This nuisance makes the computational efficiency in the solution of the global system matrix equation to greatly be degraded when such nonsymmetric BEM stiffness matrix is assembled with the symmetric and banded FEM stiffness matrix. For overcoming this shortcoming, many researchers have made their efforts and proposed various techniques. The basic idea of coupling of boundary element and finite element methods for solid mechanics goes back to [11] who combined the variational principles and the integral equation to obtain the symmetric BE stiffness matrix for computing the scalar potential field of a C-shaped depole magnet. [1] gave the same symmetric stiffness matrix of the BE region by minimizing the square of the errors in nonsymmetric off-diagonal terms. [3] provided the theoretical background for the symmetry of the stiffness matrix of the boundary element region. [10] studied several different forms of the stiffness matrices of the boundary element region for the purpose of checking the stability of these stiffness matrices for the coupling of the boundary element and finite element methods. They claimed that unsymmetric stiffness matrix of conventional boundary element method can produce the most accurate result.

${ }^{*}$ Corresponding author. Tel.: 33-1-6933-3327; fax: 33-1-6933-3026; e-mail: bonnet@lms.polytechnique.fr 
[7] provided a method for coupling of boundary element and finite element method which not only solves the equations with a symmetric matrix, but also considers the influence of unsymmetry of the boundary element region's stiffness matrix. This method was also extended to two dimensional elastoplastic problem [6].

In this paper the basic idea proposed by [6] is adopted to investigate elastoplastic problems. The elastic region is discretized by BEM, but the elastoplastic one done by the FEM. In order to improve computational efficiency, all degrees of freedom related to the BE region, except those degrees of freedom associated with interface, are condensed. The condensed stiffness matrix is equivalent to the sum of the two parts which are symmetric and antisymmetric matrices, respectively. The symmetric part is assembled into the stiffness matrix of the FEM, but antisymmetric part is taken as the corresponding influential load matrix. During the solution process of the FEM, the elastoplastic iterations which includes the influence of the antisymmetry of the condensed stiffness matrix are being carried out until the convergent results are obtained. Numerical examples are compared with corresponding results based on symmetry of the condensed unsymmetric stiffness matrix (here, we call it as the usual FE-BE method) for the interface of the boundary element region, without considering the influence of antisymmetry of interface stiffness matrix, and with the finite element solutions.

\section{Boundary element formulation}

The boundary integral equation for the analysis of an elastic body without body forces can be expressed as (see, for example, [2]):

for plane problems,

$$
C_{\alpha \beta}(p) \dot{u}_{\beta}(p)=\int_{\Gamma} U_{\alpha \beta}(p ; q) \dot{t}_{\beta}(q) \mathrm{d} \Gamma(q)-\int_{\Gamma} T_{\alpha \beta}(p ; q) \dot{u}_{\beta}(q) \mathrm{d} \Gamma(q)
$$

for axisymmetric problems,

$$
C_{\alpha \beta}(p) \dot{u}_{\beta}(p) r(p)=\int_{\Gamma} U_{\alpha \beta}(p ; q) \dot{t}_{\beta}(q) r(q) d \Gamma(q)-\int_{\Gamma} T_{\alpha \beta}(p ; q) \dot{u}_{\beta}(q) r(q) d \Gamma(q)
$$

where $\alpha, \beta=1,2 ; p, q$ represents the source point and the field point on the boundary $\Gamma$ of the body, respectively; $C_{\alpha \beta}$ is the constant coefficient whose value is defined by the tangent line at the point $p ; U_{\alpha \beta}, T_{\alpha \beta}$ are the well-known singular kernels, and $\dot{u}_{\beta}, \dot{t}_{\beta}$ the displacement and traction rates, respectively; $r$ is the distance from line of symmetry to the field point or the source point.

After discretizing the boundary and using the collocation technique at each boundary node, Eqs. (1) and (2) is written as:

$$
\left[\begin{array}{ll}
\mathbf{A}_{1} & \mathbf{A}_{2} \\
\mathbf{A}_{3} & \mathbf{A}_{4}
\end{array}\right]\left\{\begin{array}{c}
\dot{\mathbf{X}}_{\mathbf{B}} \\
\mathbf{T}_{\mathbf{B}}^{\mathbf{C}}
\end{array}\right\}=\left\{\begin{array}{l}
\dot{\mathbf{f}}_{\mathbf{B}} \\
\dot{\mathbf{f}}_{\mathbf{B}}^{\mathrm{C}}
\end{array}\right\}+\left[\begin{array}{l}
\mathbf{A}_{5} \\
\mathbf{A}_{6}
\end{array}\right]\left\{\dot{\mathbf{U}}_{\mathbf{B}}^{\mathbf{C}}\right\}
$$

where $\dot{\mathbf{X}}_{\mathbf{B}}$ is the rate vector of non-interface unknowns, and $\dot{\mathbf{T}}_{\mathbf{B}}^{\mathbf{C}}$ and $\dot{\mathbf{U}}_{\mathbf{B}}^{\mathbf{C}}$ are interface traction and interface displacement rates, respectively; $\dot{\mathbf{f}}_{\mathbf{B}}$ and $\dot{\mathbf{f}}_{\mathbf{B}}^{\mathbf{C}}$ are the part of the right hand side vector in Eq. (3), which contains the contribution of the known values.

The Eq. (3) may further be transformed into the following form by partly eliminating $\mathbf{X}_{\mathbf{B}}$ :

$$
\left[\begin{array}{ll}
\mathbf{A}_{1} & \mathbf{A}_{2} \\
\mathbf{0} & \overline{\mathbf{A}}_{\mathbf{4}}
\end{array}\right]\left\{\begin{array}{c}
\dot{\mathbf{X}}_{\mathbf{B}} \\
\dot{\mathbf{T}}_{\mathbf{B}}^{\mathbf{C}}
\end{array}\right\}=\left\{\begin{array}{c}
\dot{\mathbf{f}}_{\mathbf{B}} \\
\dot{\overline{\mathbf{f}}}_{\mathbf{B}}^{\mathbf{C}}
\end{array}\right\}+\left[\begin{array}{c}
\mathbf{A}_{5} \\
\overline{\mathbf{A}}_{\mathbf{6}}
\end{array}\right]\left\{\dot{\mathbf{U}}_{\mathbf{B}}^{\mathbf{C}}\right\}
$$

So, the equation concerning interface boundary is extracted as:

$$
\left[\overline{\mathbf{A}}_{\mathbf{4}}\right]\left\{\dot{\mathbf{T}}_{\mathbf{B}}^{\mathbf{C}}\right\}=\left\{\dot{\overline{\mathbf{f}}}_{\mathbf{B}}^{\mathrm{C}}\right\}+\left[\overline{\mathbf{A}}_{\mathbf{6}}\right]\left\{\dot{\mathbf{U}}_{\mathbf{B}}^{\mathbf{C}}\right\}
$$




\section{Finite element equations}

Following the conventional method [8], the finite element analysis for elastoplastic problems produces the following equation:

$$
\mathbf{K} \dot{\mathbf{u}}=\dot{\mathbf{f}}
$$

where $\mathbf{K}$ is the stiffness matrix, $\dot{\mathbf{u}}$ is displacement rate vector and $\dot{\mathbf{f}}$ the applied force rate vector. Eq. (6) can be written in patitioned form as follows:

$$
\left[\begin{array}{ll}
\mathbf{K}_{11} & \mathbf{K}_{12} \\
\mathbf{K}_{21} & \mathbf{K}_{22}
\end{array}\right]\left\{\begin{array}{c}
\dot{\mathbf{U}}_{\mathbf{F}} \\
\dot{\mathbf{U}}_{\mathbf{F}}^{\mathbf{C}}
\end{array}\right\}=\left\{\begin{array}{c}
\dot{\mathbf{F}}_{\mathbf{F}} \\
\dot{\mathbf{F}}_{\mathbf{F}}^{\mathbf{C}}
\end{array}\right\}
$$

where $\dot{\mathbf{U}}_{\mathbf{F}}$ and $\dot{\mathbf{U}}_{\mathbf{F}}^{\mathbf{C}}$ denotes the rate vectors of non-interface and interface displacements, respectively. $\dot{\mathbf{F}}_{\mathbf{F}}$ and $\dot{\mathbf{F}}_{\mathbf{F}}^{\mathbf{C}}$ are the associated load vectors.

\section{Stiffness matrix of the coupled BE and FE discretizations}

In order to couple Eqs. (5) and (7) to form the final equation, one can reduce Eq. (5) to a finite elements form by inverting $\overline{\mathbf{A}}_{4}$, i.e.

$$
\left[\overline{\mathbf{A}}_{\mathbf{4}}\right]^{-1}\left[\overline{\mathbf{A}}_{\mathbf{6}}\right]\left\{\dot{\mathbf{U}}_{\mathbf{B}}^{\mathbf{C}}\right\}=\left\{\dot{\mathbf{T}}_{\mathbf{B}}^{\mathbf{C}}\right\}-\left[\overline{\mathbf{A}}_{\mathbf{4}}\right]^{-1}\left\{\dot{\overline{\mathbf{f}}}_{\mathbf{B}}^{\mathbf{C}}\right\}
$$

The boundary tractions in Eq. (8), $\dot{\mathbf{T}}_{\mathbf{B}}^{\mathbf{C}}$, can be converted into the equivalent nodal force, $\dot{\mathbf{F}}_{\mathbf{B}}^{\mathbf{C}}$, of the type used in finite elements by weighting the boundary tractions with the interpolation functions used for the displacements. Therefore, $\dot{\mathbf{F}}_{\mathbf{B}}^{\mathbf{C}}$ can be expressed in the form:

$$
\left\{\dot{\mathbf{F}}_{\mathbf{B}}^{\mathbf{C}}\right\}=[\mathbf{M}]\left\{\dot{\mathbf{T}}_{\mathbf{B}}^{\mathbf{C}}\right\}=\left[\mathbf{K}_{\mathbf{B}}^{\mathbf{C}}\right]\left\{\dot{\mathbf{U}}_{\mathbf{B}}^{\mathbf{C}}\right\}+\left\{\dot{\mathbf{F}}_{\mathbf{B}}^{\mathbf{C}}\right\}
$$

where $\mathbf{M}$ is the converting matrix, $\mathbf{K}_{\mathbf{B}}^{\mathbf{C}}$, i.e. $[\mathbf{M}]\left[\overline{\mathbf{A}}_{\mathbf{4}}\right]^{-1}\left[\overline{\mathbf{A}}_{\mathbf{6}}\right]$, denotes a stiffness matrix of the BE part, $\dot{\overline{\mathbf{F}}}_{\mathbf{B}}^{\mathbf{C}}$, i.e. $[\mathbf{M}]\left[\overline{\mathbf{A}}_{\mathbf{4}}\right]^{-1} \overline{\mathbf{f}}_{\mathbf{B}}^{\mathbf{C}}$, follows from the specified displacements on the displacement boundary and tractions on the tractions on the traction boundary.

Along the common interface, displacement compatibility and force equilibrium must be satisfied. Thus, the final coupled equation will be of the form:

$$
\left[\begin{array}{cc}
\mathbf{K}_{11} & \mathbf{K}_{12} \\
\mathbf{K}_{21} & \mathbf{K}_{22}+\mathbf{K}_{\mathbf{B}}^{\mathrm{C}}
\end{array}\right]\left\{\begin{array}{c}
\dot{\mathbf{U}}_{\mathbf{F}} \\
\dot{\mathbf{U}}_{\mathbf{F}}^{\mathrm{C}}
\end{array}\right\}=\left\{\begin{array}{c}
\dot{\mathbf{F}}_{\mathbf{F}} \\
-\dot{\mathbf{F}}_{\mathbf{B}}^{\mathbf{C}}
\end{array}\right\}
$$

The matrix $\mathbf{K}_{\mathbf{B}}^{\mathbf{C}}$, which obtained form the BE part, is fully populated and asymmetric. Thus, it destroyed the sparsity of the final stiffness matrix, shown in Eq. (7). To overcome this shortcoming, $\left[\mathbf{K}_{\mathbf{B}}^{\mathbf{C}}\right]$ can be converted into the sum of a symmetric matrix $\left[\mathbf{K}_{\mathbf{B}}^{\mathbf{C}}\right]^{\mathbf{I}}$ and an antisymmetric matrix $\left[\mathbf{K}_{\mathbf{B}}^{\mathbf{C}}\right]^{\mathbf{I I}}$, i.e.

$$
\left[\mathbf{K}_{\mathbf{B}}^{\mathbf{C}}\right]=\left[\mathbf{K}_{\mathbf{B}}^{\mathbf{C}}\right]^{I}+\left[\mathbf{K}_{\mathbf{B}}^{\mathbf{C}}\right]^{I I}
$$

where

$$
\left[\mathbf{K}_{B}^{C}\right]^{I}=\frac{\left[\mathbf{K}_{B}^{C}\right]+\left[\mathbf{K}_{B}^{C}\right]^{T}}{2},\left[\mathbf{K}_{B}^{C}\right]^{I I}=\frac{\left[\mathbf{K}_{B}^{C}\right]-\left[\mathbf{K}_{B}^{C}\right]^{T}}{2}
$$

Substituing Eq. (11) into Eq. (10), one can obtain the following equation:

$$
\left[\begin{array}{cc}
\mathbf{K}_{11} & \mathbf{K}_{\mathbf{1 2}} \\
\mathbf{K}_{21} & \mathbf{K}_{\mathbf{2 2}}+\left[\mathbf{K}_{\mathbf{B}}^{\mathbf{C}}\right]^{\mathbf{I}}
\end{array}\right]\left\{\begin{array}{c}
\dot{\mathbf{U}}_{\mathbf{F}} \\
\dot{\mathbf{U}}_{\mathbf{F}}^{\mathrm{C}}
\end{array}\right\}=\left\{\begin{array}{c}
\dot{\mathbf{F}}_{\mathbf{F}} \\
-\dot{\mathbf{F}}_{\mathrm{B}}^{C}
\end{array}\right\}+\left\{\begin{array}{c}
\mathbf{0} \\
{\left[\mathbf{K}_{\mathbf{B}}^{\mathbf{C}}\right]^{\mathbf{I I}} \dot{\mathbf{U}}_{\mathbf{B}}^{\mathrm{C}}}
\end{array}\right\}
$$

If the second vector of the right hand side in the above equation is canceled, the Eq. (12) is completely equivalent to the usual coupling equation (see, for example, [1]) in which an antisymmetric matrix is not included. The present equation can be obtained by the following iterative method: 


$$
\left[\begin{array}{cc}
\mathbf{K}_{11} & \mathbf{K}_{12} \\
\mathbf{K}_{21} & \mathbf{K}_{22}+\left[\mathbf{K}_{\mathbf{B}}^{\mathbf{C}}\right]
\end{array}\right]\left\{\begin{array}{c}
\dot{\mathbf{U}}_{\mathbf{F}} \\
\dot{\mathbf{U}}_{\mathbf{F}}^{\mathbf{C}}
\end{array}\right\}^{n+1}=\left\{\begin{array}{l}
\dot{\mathbf{F}}_{\mathbf{F}} \\
\dot{-}_{\mathbf{F}}^{\mathbf{C}}
\end{array}\right\}+\left\{\begin{array}{c}
\mathbf{0} \\
{\left[\mathbf{K}_{\mathbf{B}}^{\mathbf{C}}\right]^{\mathbf{I I}} \dot{\mathbf{U}}_{\mathbf{F}}^{\mathbf{C}}}
\end{array}\right\}^{n}
$$

When $n=0, \dot{\mathbf{U}}_{\mathbf{F}}^{\mathbf{C}(\mathbf{0})}$ is chosen as zero vector.

The Eq. (13) considers the influence of the antisymmetric matrix from the interface part of BE region. In numerical computation, the iterative process with a new right hand side load may be carried out until the convegent solution is obtained. The convegent criterion is similar to equation (3.21) from [8]. In this paper, one percent is found to be a good value for tolerance of the convegent criterion.

Here, it should be mentioned that the $\left[\mathbf{K}_{\mathbf{B}}^{\mathbf{C}}\right]^{\mathbf{I}}$ in Refs. [6] and [7], in which the condensation technique did not be considered and only plane problem was studied, came from the global stiffness matrix of BE region. This means that the size of the Eq. (13) ties in with the size of all BE area, not only interface part, representing an increase of the computational time.

\section{Numerical examples}

\subsection{Infinitely long thick cylinder under internal pressure}

Infinitely long thick cylinder under internal pressure is presented to demonstrate the validity of the present algorithm and the results are compared for stresses with results obtained using analytical solution and the usual coupled FE-BE methods.

In this example, the geometrical and material data are taken to be: $R 1=100 \mathrm{~m}, R 2=200 \mathrm{~m}$, elastic modulus $E=20.58 \times 10^{4} \mathrm{MPa}$, Poisson's ratio $v=0.3$, uniaxial yields stress $\sigma_{s}=24.0 \mathrm{MPa}$, strain hardening parameter $H^{\prime}=0.0$ and the internal pressure $p$ is $14.0 \mathrm{MPa}$.

In numerical analysis, only a finite part of the cylinder with a height of $2 H=20 \mathrm{~m}$ is considered. This leads to the corresponding computational model shown in Fig. 1. The FE-BE meshes employed in the calculation are shown in Fig. 2. The results for the stress distributions are given in Figs. 3 and 4, respectively, and they are compared with the analytical solutions [4]. One can note that the numerical results from the symmetric-iterative FE-BE method have been in good agreement with analytical solution.

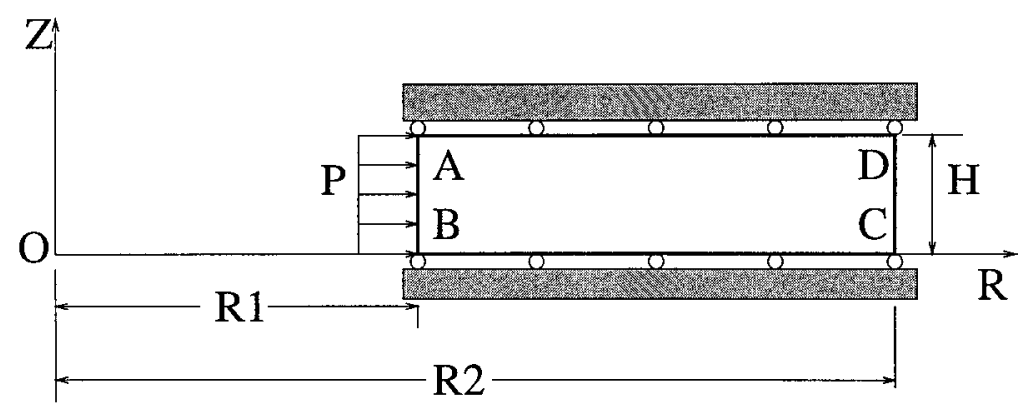

Fig. 1. Axisymmetric computational model of the thick cylinder.

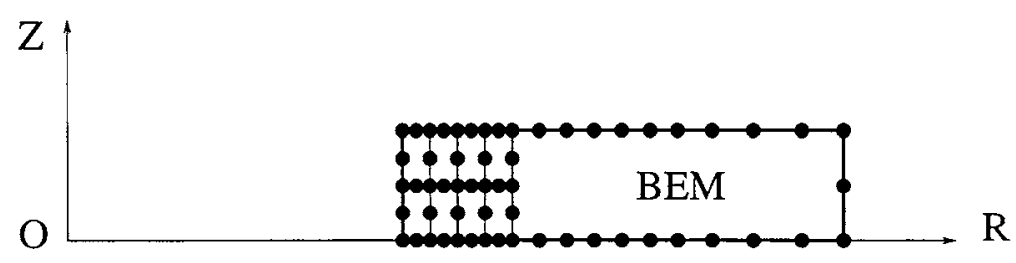

Fig. 2. Discretizations of finite element and boundary element areas. 

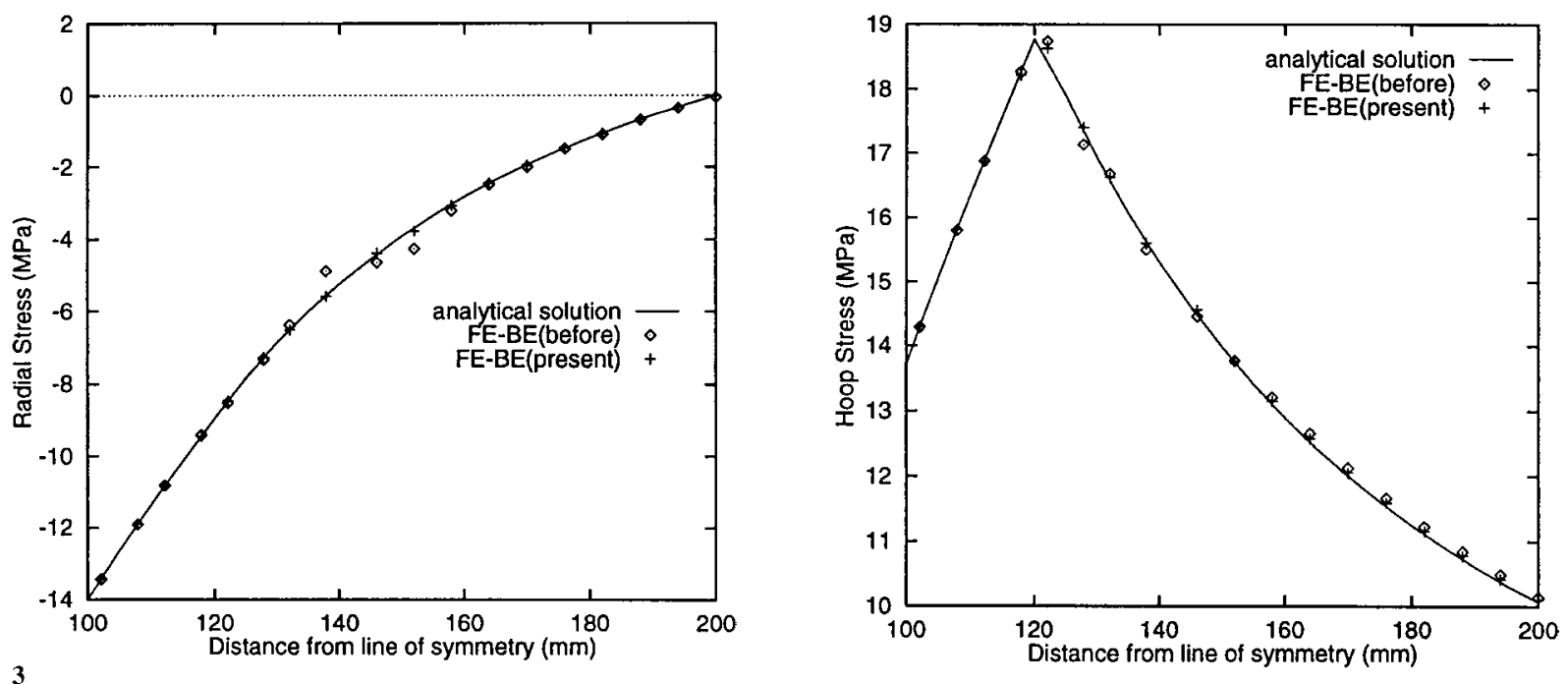

4

Fig. 3. Radial stress distributions for infinitely long thick cylinder.

Fig. 4. Hoop stress distributions for infinitely long thick cylinder.

\subsection{Thick hollow sphere}

A thick hollow sphere (Fig. 5) with internal pressure load is investigated. The geometrical and material data are taken as the following dimensionless parameters: $R 1=5, R 2=10$, elastic modulus $E=21000$ $\mathrm{MPa}$, Poisson's ratio $v=0.3$, uniaxial yields stress $\sigma_{s}=50$, strain hardening parameter $H^{\prime}=0.0$ and the internal pressure $p$ is 50 .

The meshes (FE: 36 8-node quadrilateral elements; BE: 20 3-node boundary elements) employed in the coupled FE-BE calculation are shown in Fig. 6. The numerical results together with the analytical solution are shown in Figs. 7 and 8, respectively. One again note that the usual FE-BE method exists a large dis-

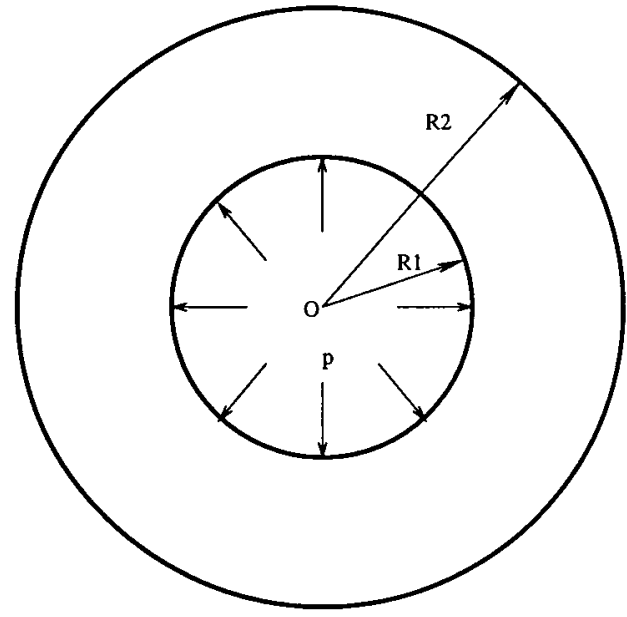

5

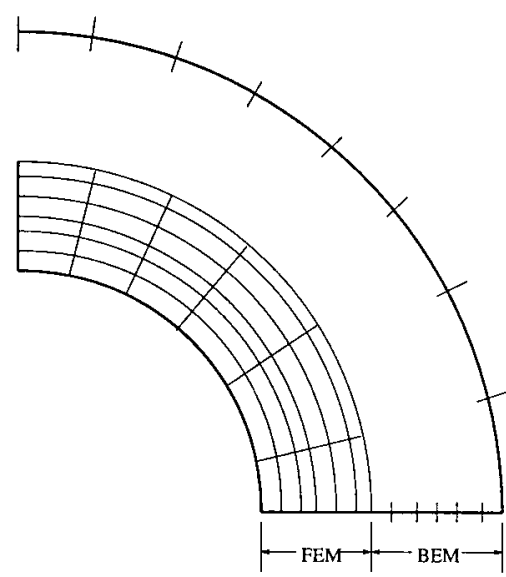

6

Fig. 5. Thick hollow sphere.

Fig. 6. Discretizations of finite element and boundary element areas. 


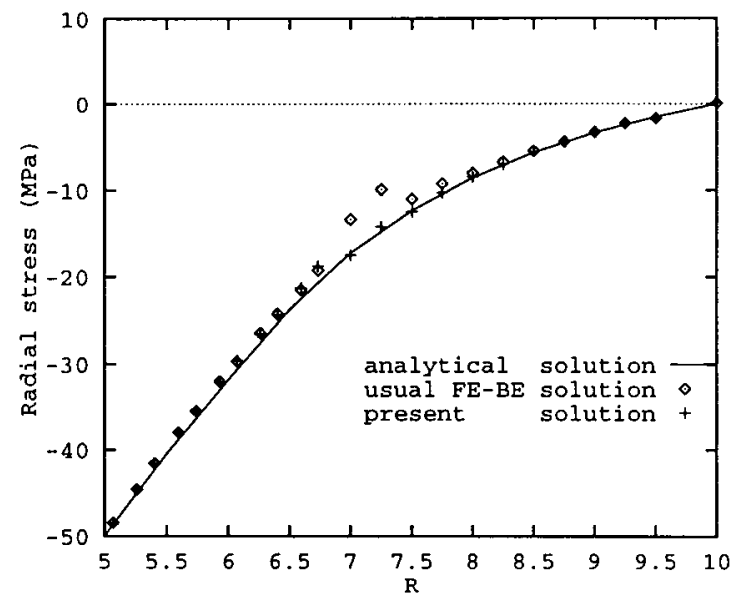

7

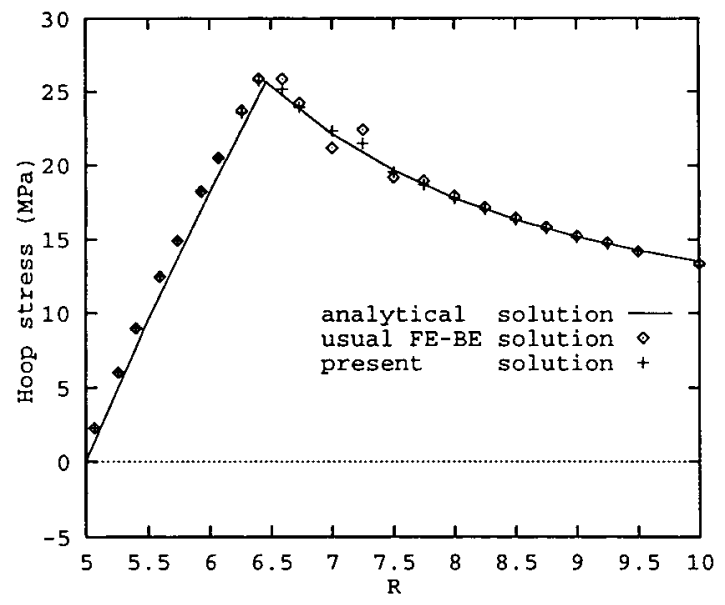

Fig. 7. Radial stress distributions for thick hollow sphere.

Fig. 8. Hoop stress distributions for thick hollow sphere.

crepancy with analytical solution [5], especially in the vicinity of the interface of FE and BE domains. Conversely, the results from present method are in good agreement with analytical solutions.

\subsection{A perforated plate in tension}

A rectangular plate of dimensions $36 \times 18 \mathrm{~mm}$ with a hole of radius $10 \mathrm{~mm}$ at the center is investigated here. Due to symmetry, only a quarter of the plate (Fig. 9) needs to be considered. The plate is assigned the following material properties: modulus of elasticity, $\mathrm{E}=7000 \mathrm{~N} / \mathrm{mm}^{2}$; Poisson's ratio, $v=0.3$; uniaxial yield stress, $\sigma_{s}=24.3 \mathrm{~N} / \mathrm{mm}^{2}$; work hardening parameter, $H^{\prime}=0.0$.

The FE and combined FE-BE models of the quarter plate are shown in Figs. 10 and 11, respectively. A distributed load of $\mathrm{p}=24.3 \mathrm{~N} \mathrm{~mm}^{2}$ was applied to the smaller sides and four load increments were used to take the load factor up to 0.55 times the applied load. The obtained results about iterative steps are given in Table 1. One notice that iterative step from present method is the same as usual FE-BE method. Fig. 12 shows the distribution of the stress $\sigma_{x}$ along dashed line, which crosses Gauss points of each element, in Fig. 11 for load factor 0.4. One can find that the present method gives a reasonable result in the near of interface of FE and BE domains, but usual FE-BE method has a discrepancy. Fig. 13 shows the development of the plastic zones with increasing value of the applied load.

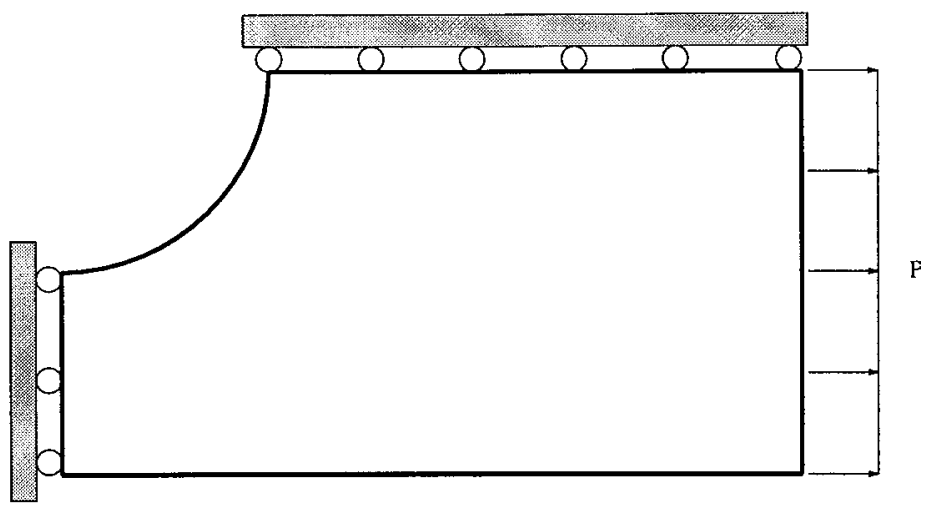

Fig. 9. A perforated plate in tension. 


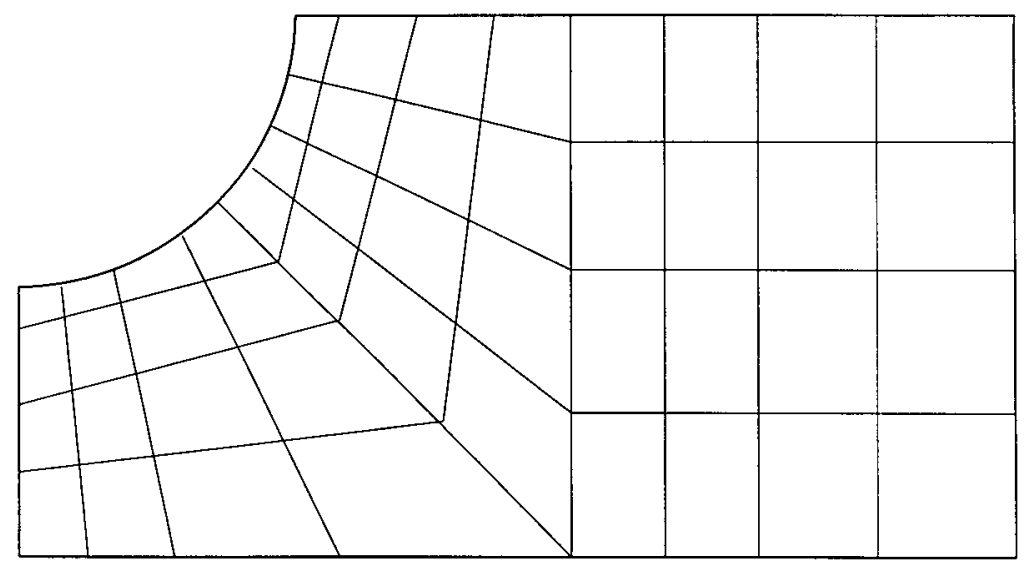

Fig. 10. FEM mesh.

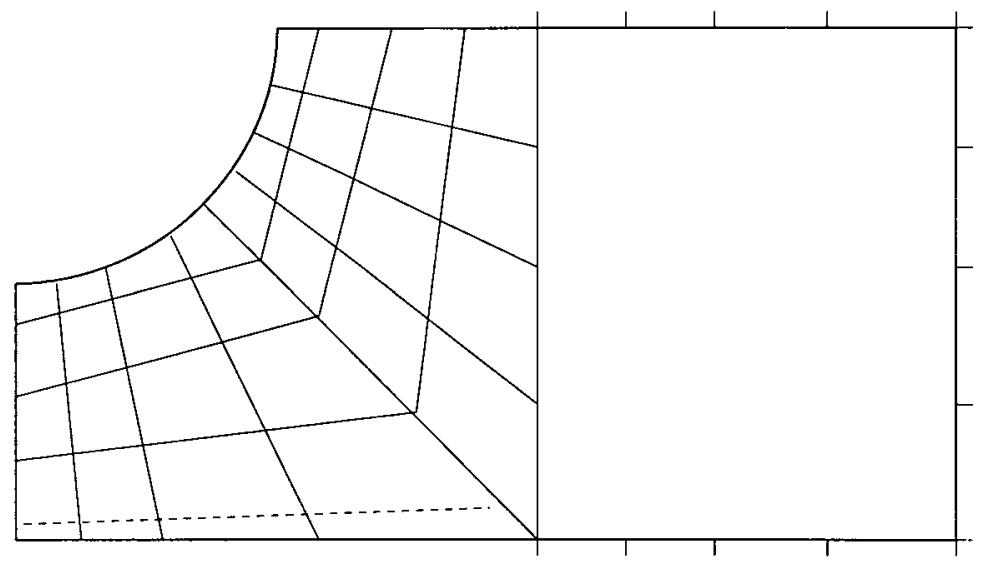

Fig. 11. FE-BE mesh.

Table 1

Iterative steps for usual FE-BE method and present method

\begin{tabular}{lll}
\hline load factor & usual Fe-BE method & present method \\
\hline 0.40 & 21 & 21 \\
0.45 & 31 & 31 \\
0.50 & 42 & 42 \\
0.55 & 68 & 68 \\
\hline
\end{tabular}

\subsection{Notched plate}

The example shown in Fig. 14 represents a notched plate subjected to a tension in the longer direction at the smaller sides. The geometrical dimensions are assumed to be: $\mathrm{L}=18 \mathrm{~mm}, \mathrm{~W}=10 \mathrm{~mm}, \mathrm{C}=5 \mathrm{~mm}$, $\alpha=45^{\circ}$. The material parameters are the same as the above example. The finite element and combined FEBE meshes are illustrated in Figs. 15 and 16, respectively. Five load increments were taken as load factor values up to 0.7 times the applied load of $24.3 \mathrm{~N} / \mathrm{mm}^{2}$. Fig. 17 shows the development of plastic zones with increasing loading. The iterative steps needed from usual FE-BE method and present method are given in Table 2. Figs. 18 and 19 shows the distribution of the stress $\sigma_{x}$ along dashed line, which crosses Gauss points of each element, in Fig. 15 for load factor 0.55 and 0.65 , respectively. The same conclusion with the above example was obtained. 


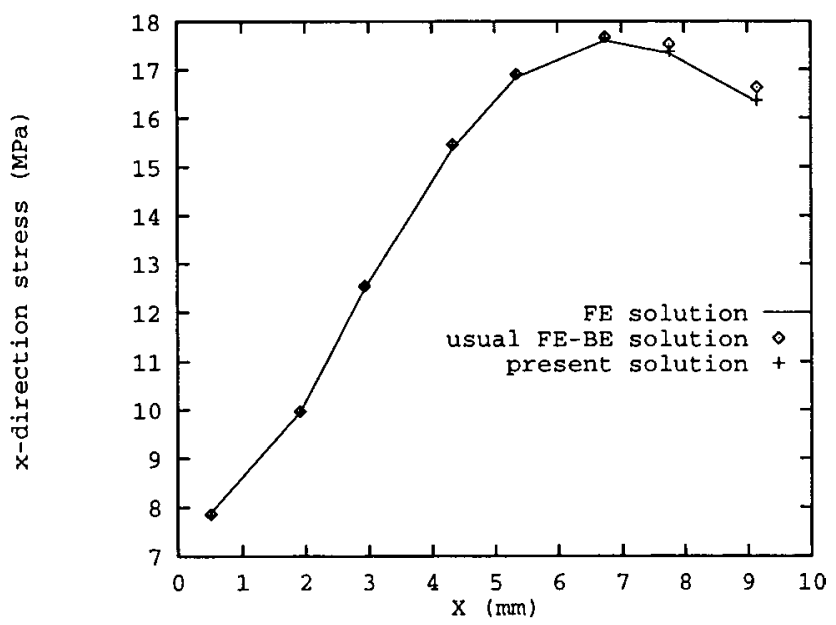

Fig. 12. Stress distribution of $x$-direction along dashed line shown in Fig. 11.

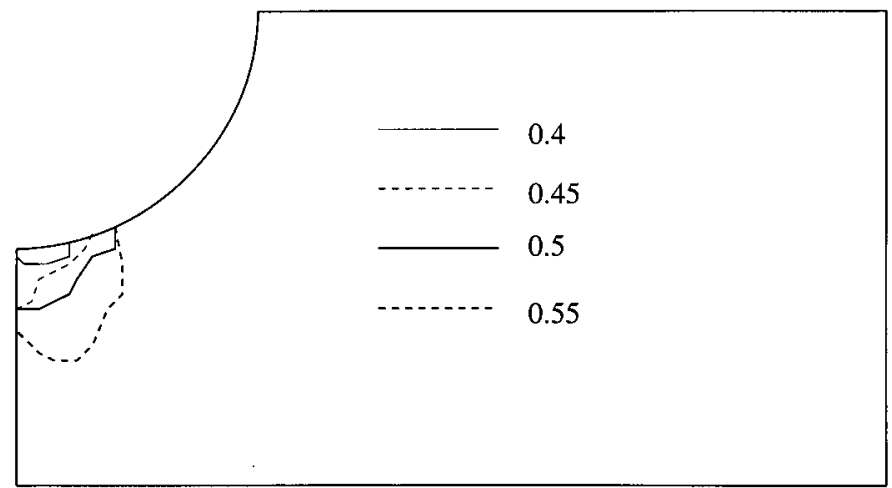

Fig. 13. Development of plastic zones with increasing loading.

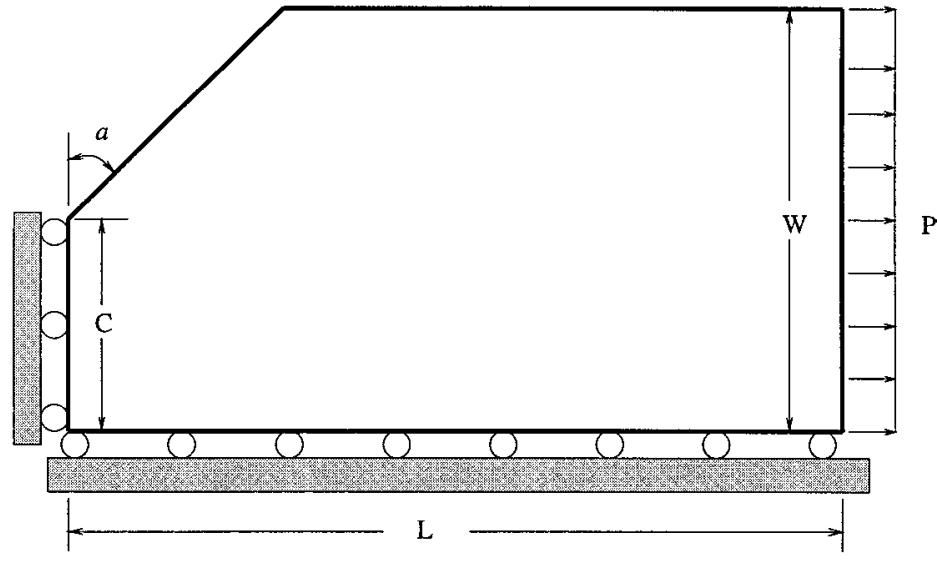

Fig. 14. Notched plate in tension.

\subsection{Centre cracked plate}

The problem considered (Fig. 20) is that of a plate under tension which contains a crack of length $2 \mathrm{a}$ perpendicular to the direction of loading. The width of the plate is $2 \mathrm{~W}$ and the thickness assumed to be 


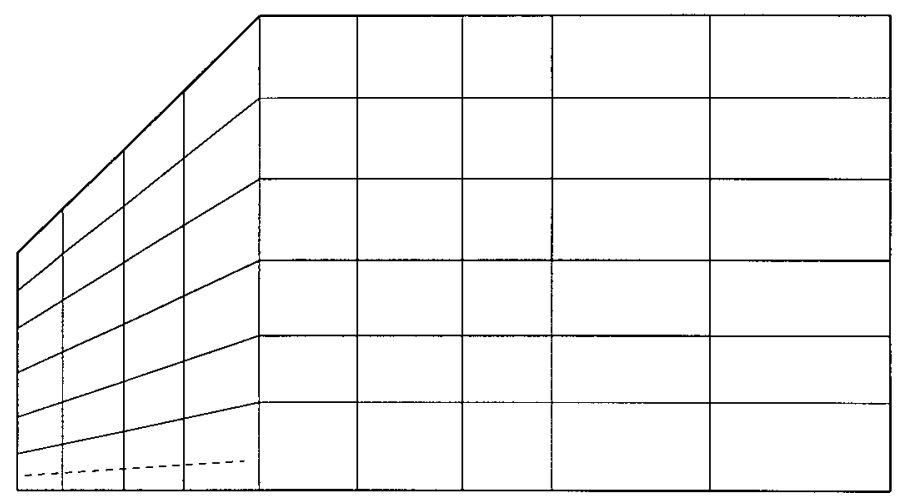

Fig. 15. FEM mesh.

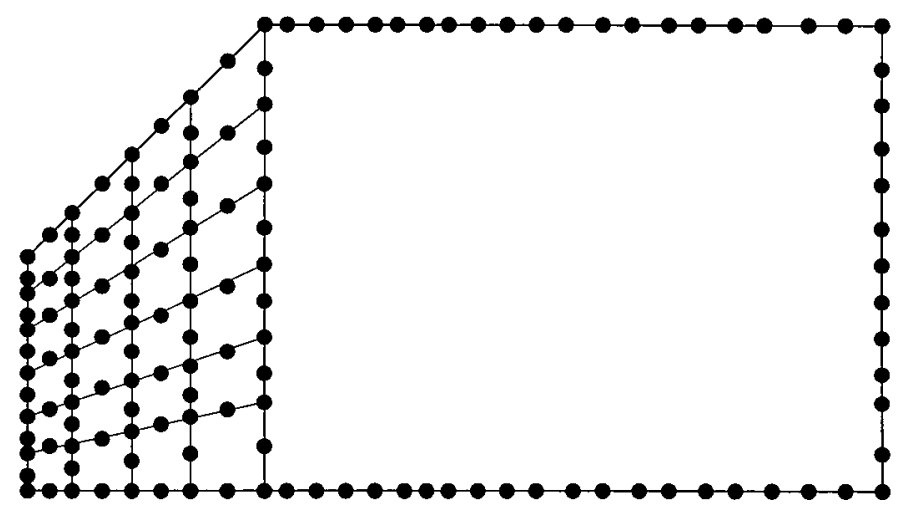

Fig. 16. FE-BE mesh.

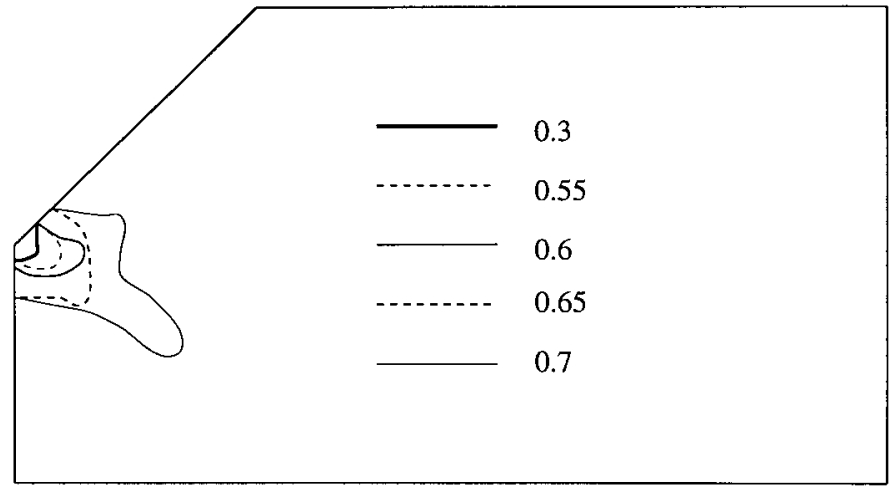

Fig. 17. Development of plastic zones with increasing loading.

Table 2

Iteratives steps for usual FE-BE method and present method

\begin{tabular}{lll}
\hline load factor & usual Fe-BE method & present method \\
\hline 0.30 & 3 & 3 \\
0.55 & 4 & 4 \\
0.60 & 4 & 4 \\
0.65 & 5 & 5 \\
0.70 & 5 & 5 \\
\hline
\end{tabular}




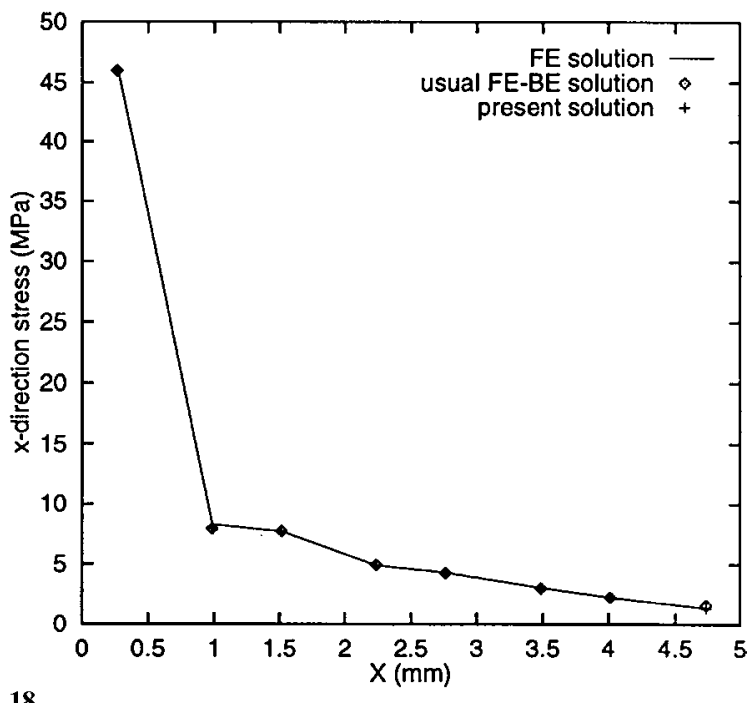

18

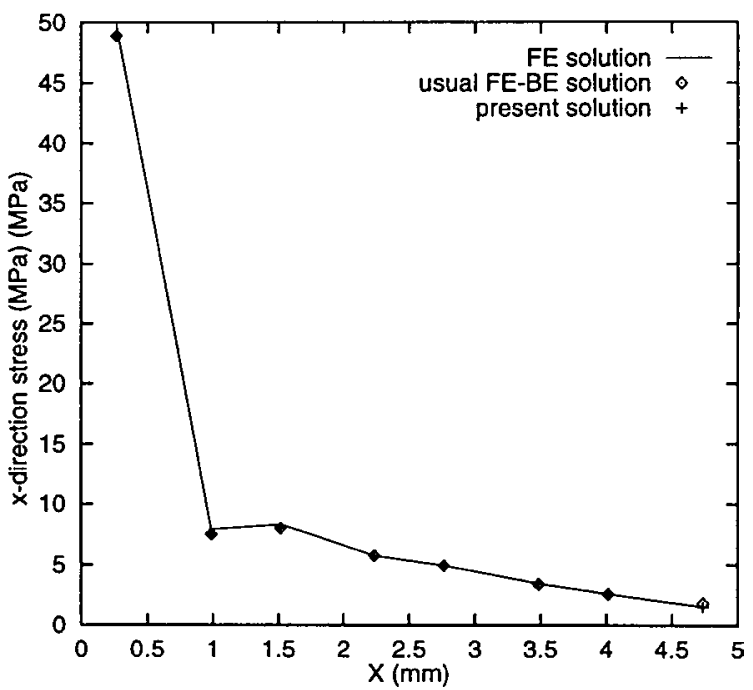

19

Fig. 18. Stress distribution of $x$-direction for load factor 0.55 .

Fig. 19. Stress distribution of $x$-direction for load factor 0.65 .

unity. The length of the strip was assumed to be $5 \mathrm{~W}$. The initial crack length was chosen as a $=0.4 \mathrm{~W}$. The material properties assumed for analysis are taken as the following dimensionless data: modulus of elasticity, $\mathrm{E}=1000$; Poisson ratio, $v=0.3$; uniaxial yield stress $\sigma_{s}=100$; strain harding parameter $H^{\prime}=0.0$. Three load increments was used as load factors up to 0.6 times the applied load of $p=100$.

Fig. 21 shows the FE mesh consisting of 20 isoparametric quadratic elements and the $\mathbf{J}$ integral paths used in the analysis of elastoplastic problem. The combined FE-BE mesh with 18 isoparametric finite elements and 14 isoparametric boundary elements, respectively, is shown in Fig. 22. J-integrals obtained by FEM [9], the usual FE-BE method and present method are given in Fig. 23. It can be observed from Fig. 23 that J-integrals from present method and usual FE-BE method are in good agreement with the FE results of [9]. Discrepancy between J-integrals from present method and usual FE-BE method is quite small. The reason is that $\mathrm{J}$-integral paths were taken as those close to crack tip, not near interface between FE and BE parts. The development of yield surface with the increasing load is illustrated in Fig. 24.

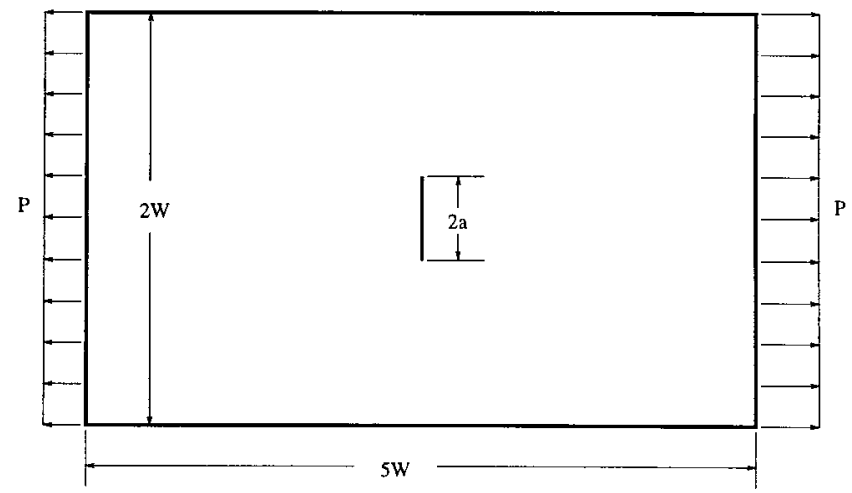

Fig. 20. Cracked plate. 


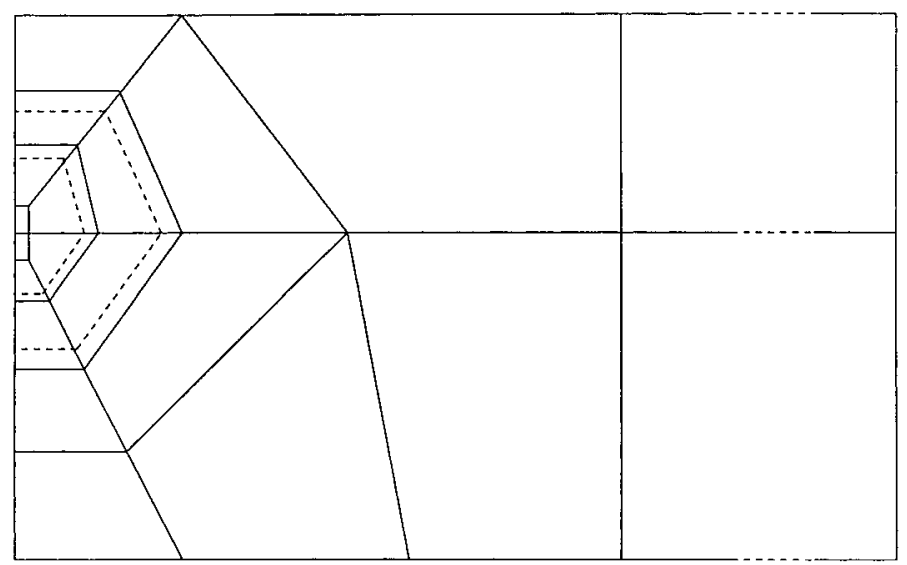

Fig. 21. FE mesh.

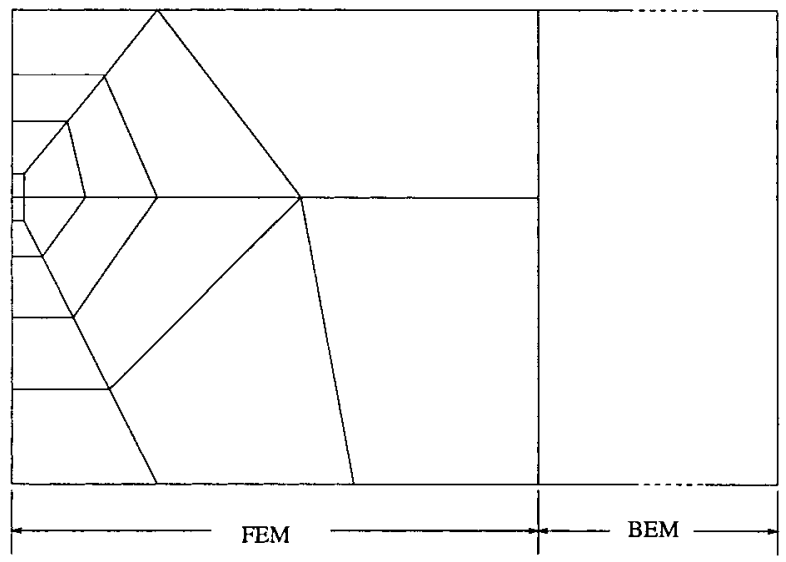

Fig. 22. FE-BE areas.
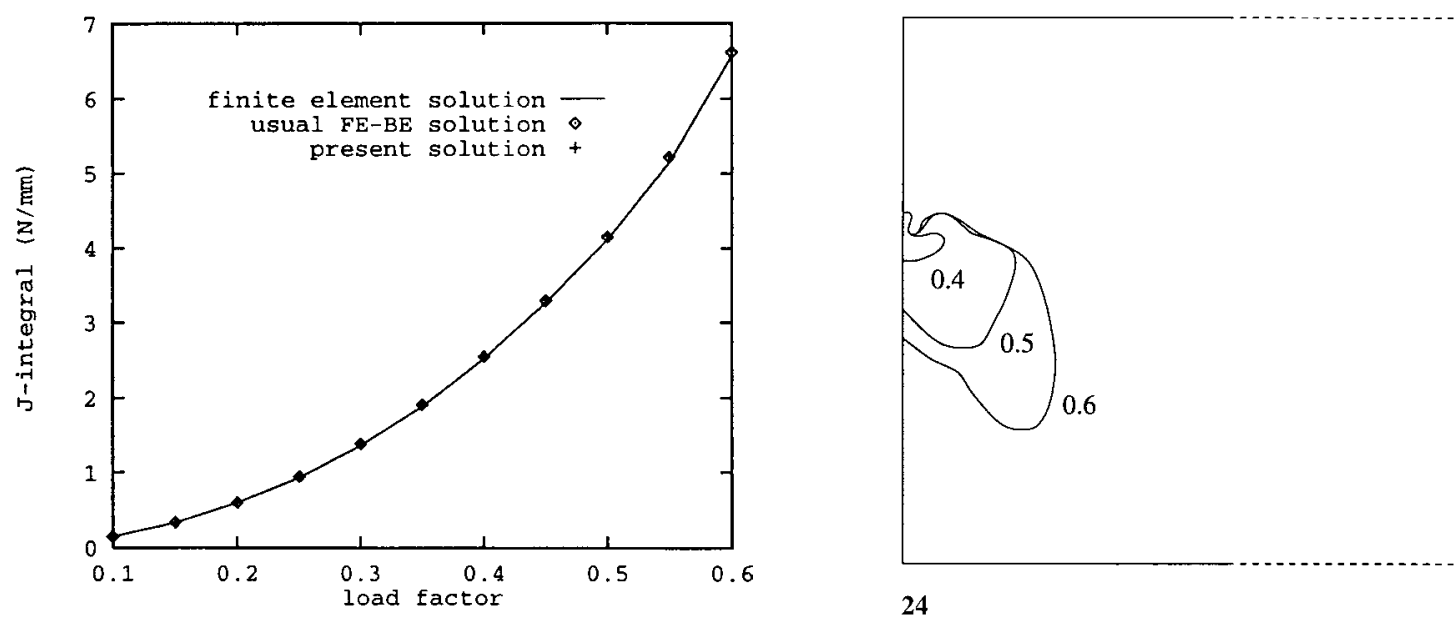

23

Fig. 23. J-integrals for several load factors.

Fig. 24. Iterative steps for usual FE-BE method and present method. 


\section{Conclusions}

The main conclusion drawn are as follows:

1. Symmetric-Iterative FE-BE coupling method is extended to solve the axisymmetrically elastoplastic and plane fracture problems;

2. The obtained results are in good agreement with analytical solution or corresponding FEM solutions;

3. In the near of interface of FE and $\mathrm{BE}$ areas, present method can give a better result than usual FE-BE method;

4. The present method is more efficient than solving for the unsymmetric matrices because only half of the matrix is operated upon during Gauss elimination;

5. Present method can be extended to three dimensional elastoplastic, elasto-viscoplastic, soil-structure interaction and other associated areas.

\section{References}

[1] C.A. Brebbia, P. Georgiou, Combination of boundary and finite elements for elastostatics, Appl. Math. Modelling 3 (1979) $212-220$.

[2] C.Y. Dong, Some basic aspects on the boundary element methods of elastoplasticity and their applications on contact problems, Ph.D Thesis, Tsinghua University, 1992.

[3] F. Hartman, The derivation of stiffness matrices from integral equations, in: C.A. Brebbia Ed., Boundary Element Methods Springer, Berlin, 1981.

[4] P.G. Hodge, G.N. White, A quantitative comparison of flow and deformation theories of plasticity, J. Appl. Mech. 17 (1950) $180-184$.

[5] J.H. Kane, Boundary Element Analysis in Engineering Continuum Mechanics, Prentice Hall, London, 1994.

[6] J.M. Ma, G. Yagawa, T.A. Cruse Eds, A coupling method of boundary element and finite element for elastoplastics, S.N. Atluri. Computational Mechanics'95, Springer, Berlin, 1995.

[7] J.M. Ma, M.F. Le, A new method for coupling of boundary element method and finite element method, Appl. Math. Modelling 16 (1992) 43-46.

[8] D.R. Owen, E. Hinton, Finite Elements in Plasticity: Theory and Practice, Pineridge Press, Swansea, UK, 1980.

[9] D.R. Owen, A.J. Fawkes, Engineering Fracture Mechanics: Numerical Methods and Applications, Pineridge Press, Swansea, UK, 1983.

[10] O. Tullberg, L. Bolteus, A critical study of different boundary element stiffness matrices, in: C.A. Brebbia (Ed.), Boundary Element Methods in Engineering, Springer, Berlin, 1982.

[11] O.C. Zienkiewicz, D.W. Kelly, P. Bettess, The coupling of the finite element method and boundary solution procedures, Int. J. Num. Meth. in Eng. 11 (1977) 355-375. 